\title{
A QSO survey via optical variability and zero proper motion in the M 92 field
}

\section{Follow-up spectroscopy and properties of the QSO sample}

\author{
H. Meusinger^ and J. Brunzendorf ${ }^{\star}$
}

\begin{abstract}
Thüringer Landessternwarte Tautenburg, 07778 Tautenburg, Germany
\end{abstract}
e-mail: meus@tls-tautenburg.de, brunz@tls-tautenburg.de

Received 10 April 2001 / Accepted 30 May 2001

\begin{abstract}
The combination of variability and proper motion constraints (VPM search) is believed to provide an efficient yet unconventional search strategy for QSOs with selection effects quite different from conventional optical QSO surveys. We performed a VPM QSO search in a 10 square degrees field around M 92 on 162 selected digitised Schmidt plates in the Johnson $B$ band with a time-baseline of more than three decades. In Paper I, we constructed a sample of VPM QSO candidates with $B \leq 19.7$. The spectroscopic follow-up observations revealed 58 QSOs and 7 Seyfert 1 galaxies as well as 27 narrow-emission line galaxies. We present the lowresolution spectra and the data for the QSOs and Seyfert galaxies: AGN types, accurate positions, redshifts, mean $B$ magnitudes, and colour indices, along with the selection parameters of the VPM search, i.e., indices for variability, proper motion, and image structure. The redshifts cover the interval $0 \lesssim z \lesssim 3$; absolute magnitudes are in the range $-21 \lesssim M_{B} \lesssim-29$. The statistical properties of the resulting QSO sample are studied in detail. No significant differences were found between our VPM QSO sample and published QSO samples from multicolour or spectroscopic surveys. The fraction of QSOs with unusual colours is estimated to be less than 5 per cent within the magnitude range $B \leq 19.7$.
\end{abstract}

Key words. galaxies: active - galaxies: statistics - quasars: general

\section{Introduction}

The combination of selection constraints based exclusively upon variability and proper motion provides an unconventional QSO search strategy $(\mathrm{VPM}$ search $=V$ ariability and Proper $M$ otion search) that does not explicitely rely on the particular shapes of the spectral energy distributions (SEDs) of QSOs. It has been speculated that "a search for objects which are both variable and stationary is a powerful technique for efficiently finding QSOs with no selection bias with regard to colour, redshift, spectral index, or emission line equivalent widths" (Majewski et al. 1991; Véron 1993). On the other hand, it has been demonstrated by several studies, most definitely by Hook et al. (1994), that the variability of QSOs is statistically anticorrelated with luminosity. Variability-selected QSO samples might, therefore, be biased against high luminosity, and therewith high redshift QSOs. Nevertheless, the

Send offprint requests to: $\mathrm{H}$. Meusinger,

e-mail: meus@tls-tautenburg.de

* Visiting Astronomer, German-Spanish Astronomical Centre, Calar Alto, operated by the Max-Planck-Institute for Astronomy, Heidelberg, jointly with the Spanish National Comission for Astronomy.
VPM technique is of particular importance, at least as an alternative and complementary search method with selection constraints that are fundamentally different from those of more conventional search strategies. For example, Webster et al. 1995 found an excess reddening in a sample of radio-selected QSOs relative to optically selected ones (see also Drinkwater et al. 1997; Francis et al. 2000). If reddening is universal to all QSOs, conventional optical surveys can be seriously incomplete. The VPM search can contribute to clarify the important question to what extent conventional QSO surveys systematically oversee hitherto unknown and possibly substantial QSO populations. Moreover, the long-term lightcurves of a comprehensive sample of QSOs detected in such a project can provide valuable information about the statistical properties of QSO variability.

We performed VPM QSO searches on digitised Schmidt plates of two Tautenburg "standard fields". At first, a field near the North Galactic Pole was surveyed on 85 plates digitised by means of the APM facility, Cambridge (Meusinger et al. 1995; Scholz et al. 1997). Follow-up spectroscopy of the QSO candidates from this study is in progress; preliminary results were presented by Meusinger et al. (1997, 1998, 1999). 
A subsequent study, described in the present series of papers, is concerned with a field of intermediate galactic latitude $\left(b \approx 35^{\circ}\right)$, centred on the globular cluster M 92 . This field was selected mainly because of the exceptionally large number of available archival plates. The procedure applied for the M92 field is, in principle, similar to that one from the M3 field. There are, however, remarkable differences in the details: 1) lightcurves and proper motions of the objects in the M92 field were measured on twice as many plates (162 $B$ plates), 2) the plates were digitised by means of the Tautenburg Plate Scanner TPS (Brunzendorf \& Meusinger 1999), 3) the basic object sample from which QSO candidates are to be selected is defined in a different way and is much larger with 35000 objects in the magnitude range $8 \leq B \leq 20.5$ and with a 99 per cent completeness limit $\left.B_{\text {compl }}=19.8,4\right)$ variability indices are defined in such a way that they are directly related to the probability of an object to be variable.

In Paper I of this series (Brunzendorf \& Meusinger 2001), we described the observational material of the VPM search in the M92 field, the photometric and astrometric data reduction, and the selection procedure of the QSO candidates. Expected major selection effects were also discussed in Paper I. Preliminary results from follow-up spectroscopy were given in Brunzendorf \& Meusinger (2000). Here, we present the final results of the spectroscopic follow-up observations of the VPM QSO candidates from several observation campaigns and investigate the properties of the resulting QSO sample. The observations will be described in Sect. 2. The list of the detected QSOs and Seyfert 1 galaxies is presented in Sect. 3. A detailed discussion of the VPM QSO sample follows in Sect.4. Finally, the conclusions are given in Sect. 5 .

\section{Observations}

In Paper I, we defined three categories of QSO candidates from the VPM survey of the M92 field: 1) 62 candidates of high priority, 2) further 57 candidates of medium priority, and 3) further 5709 stationary star-like objects, which form the low-priority sample. Spectroscopic follow-up observations have been focussed on the high- and mediumpriority candidates. A number of low priority candidates were included as well.

For the brighter candidates, multi-object spectroscopy was performed which allows the inclusion of a relatively large number of low priority candidates. For the fainter candidates, single-object spectra were taken in the longslit mode. In both modes, we aimed at a doubtless classification of as many objects as possible. Therefore, we chose a low linear dispersion and observed the candidates up to an airmass of two. During dusk and dawn, a few spectrophotometric standard stars were observed. The key data of the observation campaigns are summarized in Table 1.

\subsection{Multi-object spectroscopy with TAUMOK}

Candidates with $V \lesssim 18$ were observed with the multiobject spectrograph TAUMOK in the Schmidt focus of the Tautenburg $2 \mathrm{~m}$ telescope in combination with a Tektronix TK1024A CCD. TAUMOK allows to obtain spectra of up to 35 objects simultaneously. In operation, 36 circularly arranged rods, controlled by a single robot, can be moved within an area of $2.3(160 \mathrm{~mm})$ diameter. The first rod carries an image bundle for guiding purposes. Each of the 35 remaining rods carries a fibre with a diameter of $100 \mu \mathrm{m}$ ( $5^{\prime \prime}$ on the sky). With additional fibres available at 17 of the rods, the sky background is simultaneously measured at a distance of $40^{\prime \prime}$ from the object fibre. The rear ends of the fibres form the entrance slit of the Zeiss spectrograph UAGS. The highest available reciprocal dispersion amounts to $400 \AA \mathrm{mm}^{-1}$. Internal spectral lamps are used for the wavelength calibration prior and/or after the observations. The robot allows to place the fibres with an accuracy of about $10 \mu \mathrm{m}$. In reality, the positions are less accurate owing to thermal and mechanical effects. Therefore, a scan mode was applied at the beginning of the observations to determine the most accurate positions. A more detailed description of TAUMOK is given by Lehmann et al. (1995) and Ball (2000). For the reduction of the TAUMOK spectra, a semi-automatized reduction software package written by Ball (2000) was applied which is based on IRAF standard procedures for multiobject spectroscopy.

\subsection{Single-object spectroscopy with CAFOS}

The fainter candidates were observed with the focal reducer and faint object spectrograph CAFOS at the $2.2 \mathrm{~m}$ telescope of the German-Spanish Astronomical Centre (DSAZ) on Calar Alto, Spain. In two observation runs in the summer of 1998 and 1999, respectively, low-resolution spectra were taken with CAFOS equipped with the B-400 grism and a SITe1d CCD. The weather conditions were good, partly photometric. Depending on the seeing (typically $\left.1 \ldots 2^{\prime \prime}\right)$, a slit width was chosen that results in a linear resolution of $22 \ldots 38 \AA$. The orientation of the slit was always North-South.

Prior to each spectroscopic observation, an acquisition exposure was taken for the accurate positioning of the long-slit. With exposure times of $60 \mathrm{~s}$, the acquisition frames reach a limiting magnitude of $R \approx 23$ and provide additional information for the morphological classification of the candidates. At a seeing of $1^{\prime \prime}$, galaxies can be discriminated from stars up to galaxy redshifts of $z \approx 0.5$. In a few cases, QSO candidates having star-like images on the Schmidt plates turned out to be close visual double stars with separations of about $1^{\prime \prime}$. The measured variability of such objects usually is of artificial nature produced by the plate-to-plate variations of the image structure and the poor fit of the applied Gaussian profiles during the photometric reduction. These objects were removed from the candidate list without taking a spectrum, since it is 
Table 1. Key data of the spectroscopic follow-up observation campaigns. 75 objects were additionally observed by chance with the CAFOS long-slit.

\begin{tabular}{lcc}
\hline telescope & Tautenburg $1.34 \mathrm{~m}$ Schmidt & Calar Alto $2.2 \mathrm{~m}$ \\
device & TAUMOK & CAFOS \\
wavelength range & $3500 \ldots 8500 \AA$ & $3500 \ldots 9000 \AA$ \\
reciprocal linear dispersion & $400 \AA \mathrm{mm}^{-1}$ & $400 \AA \mathrm{mm}^{-1}$ \\
& $=9 \AA$ per pixel & $=9 \AA$ per pixel \\
effective linear resolution & $26 \AA$ & $22 \ldots 38 \AA$ \\
\hline dates of observations & May 1998, June 1998 & July 1998, July 1999 \\
number of nights & $2 \times 15$ & $2 \times 5$ \\
effective total integration time & 15 hours & 66.5 hours \\
atmospheric transparency & alternating & good \\
total number of objects & 117 & $150+75$ \\
magnitude range & $15.4 \leq \bar{B} \leq 18.2$ & $16.9 \leq \bar{B} \leq 19.7$ \\
integration time per object & $30 \ldots 170 \mathrm{~min}$ & $5 \ldots 65 \mathrm{~min}$ \\
\hline
\end{tabular}

unlikely (yet not impossible) that such close visual binaries are formed by gravitationally lensed QSOs or QSO pairs instead by ordinary stars. For the remaining candidates, a first spectrum was taken with a typical exposure time of $300 \ldots 600 \mathrm{~s}$. A quick-look reduction of this spectrum served to estimate the required total intergration time, which varied between $5 \mathrm{~min}$ and $65 \mathrm{~min}$ and primarily depended on the strength of the emission lines and the object magnitude. The data reduction was performed under MIDAS and followed the standard procedures of the long-slit spectroscopy package. The wavelength calibration was done by means of calibration lamp spectra. We abstained from a flux-calibration simply because it is not essential for our main purposes, which are the confirmation of the QSOs and the determination of the redshifts.

\subsection{Overview of the spectroscopically observed objects}

Spectra were taken for a total of 234 VPM QSO candidates (see Table 3 in Paper I) with the following systematics:

- all 62 high-priority candidates;

- 34 out of the 57 medium-priority candidates, namely;

- all 28 objects with proper motion indices $I_{\mu} \leq 3$;

- the 6 objects having $3<I_{\mu} \leq 4.3$ and showing strong variability $\left(I_{\sigma}>3\right)$;

- 138 out of the 5709 low-priority candidates, including in particular such objects with either significant overall variability $\left(I_{\sigma}>11\right)$ or strong long-term variability $\left(I_{\Delta}>2\right)$.

A number of objects observed with TAUMOK were reobserved with CAFOS for an improved signal-to-noise ratio. In addition to the target observations, spectra of further 87 objects from the basic sample were obtained gratis, since these objects were either located by chance on the long-slit of CAFOS which has a length of $9^{\prime}$, or observed with free TAUMOK fibres. Thus, the total number of objects observed amounts to 321 .

\section{Source classification}

According to their follow-up spectra, the objects are classified into three categories: 1) broad emission line objects, 2) narrow emission line galaxies, and 3) foreground stars.

The first category comprises all objects which show one or several broad and redshifted $(z>0)$ emission lines that are broader than the forbidden lines. This category includes QSOs and Seyfert 1 galaxies, but not Seyfert 2 galaxies. All objects assigned to this class show emission lines that are characteristic of AGNs. The discrimination between QSOs and Seyfert 1s is simply based on the usual luminosity criterion: broad emission line objects with $M_{B}<-23$ are called QSOs, the fainter objects are called Seyfert $1 \mathrm{~s}$. The absolute magnitude $M_{B}$ is computed according to Eq. (22) from Paper I for $H_{0}=$ $50 \mathrm{~km} \mathrm{~s}^{-1} \mathrm{Mpc}^{-1}, q_{0}=0$, and using the $K_{B^{-c o r r e c t i o n}}$ given in Paper I. Figure 1 shows the plot of the absolute magnitudes $M_{B}$ versus $z$. For $z \leq 0.55$, all QSOs are within the magnitude limit, and most objects are classified as Seyfert 1s. For larger $z$, the QSO sample is magnitudelimited, and Seyfert $1 \mathrm{~s}$ are not detectable.

Objects with prominent redshifted $(z>0)$ narrow emission lines, but without broad emission lines, are called narrow-emission line galaxies (NELGs). Most of these NELGs appear quasi-stellar on the Schmidt plates, yet are resolved with galaxy-like morphology on the CAFOS acquisition frames. The measured redshifts cover the range $z=0.028 \ldots 0.21$. This class is expected to include in particular Seyfert 2 galaxies, LINERs, and H II galaxies.

The presence of unredshifted $(z=0)$ stellar absorption lines is taken as indicative for foreground stars. All these objects show a non-resolved image structure on the acquisition frames. At a first glance, most of the spectroscopically identified foreground stars are normal main sequence stars or giants without unusual spectral features. The remarkable exception is the strongly variable faint high-latitude carbon star J1714.9+4210 with very strong 


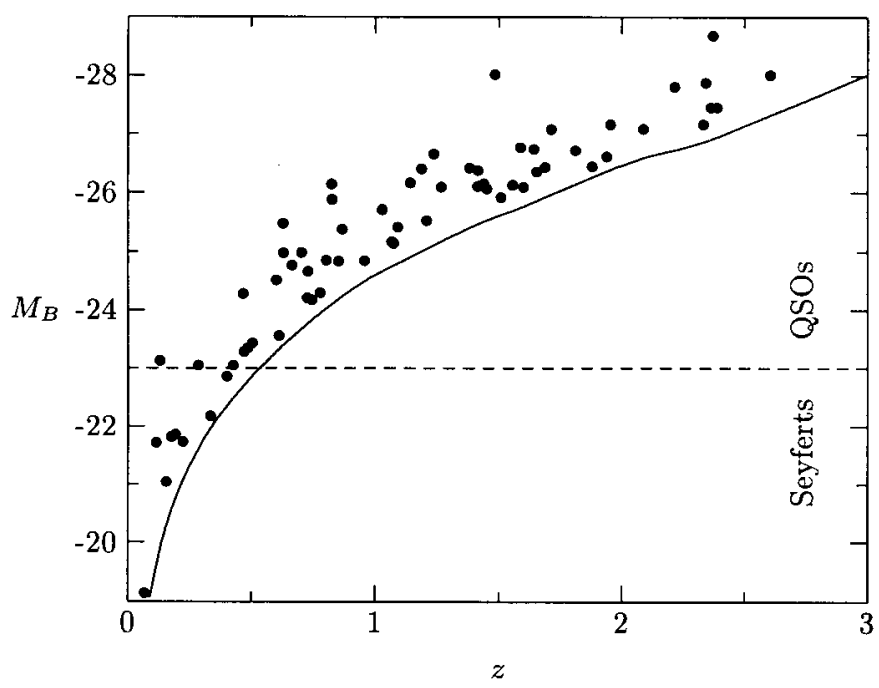

Fig. 1. Absolute magnitude $M_{B}$ of the discovered QSOs and Seyferts versus redshift $z$. The continuous line indicates the magnitude limit $B \leq 19.7$ of the survey.

Table 2. Results from the clasification of the follow-up spectra: numbers of objects in different catagories. (ETGs means earlytype galaxies.)

\begin{tabular}{lrrrrr}
\hline priority & high & medium & low & none & $\Sigma$ \\
total & 62 & 57 & 5709 & 28698 & 34526 \\
observed & 62 & 34 & 138 & 87 & 321 \\
\hline QSOs & 39 & 5 & 13 & 1 & 58 \\
Seyfert 1s & 4 & 2 & 0 & 1 & 7 \\
NELGs & 1 & 2 & 16 & 8 & 27 \\
ETGs & 0 & 0 & 3 & 0 & 3 \\
double stars & 2 & 4 & 7 & 2 & 15 \\
single stars & 16 & 21 & 99 & 75 & 211 \\
\hline
\end{tabular}

absorption bands which is probably a distant halo giant (Meusinger \& Brunzendorf 2001). The stellar content of the VPM survey will be discussed in more detail elsewhere.

Three objects could not be classified within this scheme. Their spectra do not show any emission or typical stellar absorption lines with equivalent widths $>10 \AA$. On the acquisition frames, these objects appear slightly resolved, like early-type galaxies without prominent nuclei. Two of these objects have absorption spectra consistent with early-type galaxies at $z=0.089$ and 0.123 , respectively. For the third object, the signal-to-noise ratio is too low to identify such absorption features. With regard to the low long-term variability indices, $I_{\Delta}<1.3$, it seems likely that these objets are "normal" early-type galaxies, where the measured overall-variability is artificial due to the partly resolved image structure (see the discussion of Fig. 2 in the following subsection).

The results of the classification are summarized in Table 2. In the high priority subsample, 70 per cent of the candidates were found to be QSOs or Seyfert 1s, while the contamination by foreground stars is as low as 30 per cent. The number of 44 QSOs from the combined subsam- ple of high-priority and medium-priority candidates is in a perfect agreement with the a priori estimate (Paper I) of 42 QSOs. As discussed in Paper I, about 50 further QSOs with $B \leq 19.7$ are expected to be contained in the low-priority subsample with $B$ magnitudes near the survey limit. This assumption could be verified for 13 QSOs up to now.

The low-resolution spectra for the 58 QSOs and 7 Seyfert 1 galaxies are shown in Fig. 9; the data are listed in Table 3 for the QSOs and Table 4 for the Seyfert 1s, respectively, sorted by decreasing $z$. The redshifts given there are the mean redshifts from all significant emission lines measured for a given object, where the wavelength of a single line is measured by a Gaussian approximation of the line profile using the MIDAS procedure CENTER/GAUSS.

A search with VizieR ${ }^{1}$ yields detections in other spectral ranges for 19 objects from Tables 3 and 4 . The subsamples of AGNs identified with radio or X-ray sources will be discussed in a separate study. Two QSOs (numbers 57 and 58) and one Seyfert (number 65) were found to have already $z$ measurements in the 9 th Edition of the AGN catalogue by Véron-Cetty \& Véron (2000).

One of the QSOs found (No. 58) belongs to the large subsample of objects which were actually not considered as candidates. The reason for missing this QSO in the candidate sample is its morphology index $I_{\text {nonstellar }}=6.5$, which is slightly above the chosen limit $I_{\text {nonstellar }} \leq 6$ for unresolved objects. We observed this non-candidate since its strong variability and zeroproper motion along with the nearly stellar image structure, the UV excess and the identification with the Xray source 2RXP J171503.0+442950 (ROSAT Consortium 2000) make it a high-priority candidate for a low-redshift QSO. Indeed, this candidate proved to be the QSO with the lowest redshift $(z=0.152)$ in our sample. With $M_{B}=$ -23.1 , this object is near the dividing line between QSOs and Seyfert 1s. In Paper I, we estimated the total number of QSOs which were mis-classified as non-candidates due to their non-stellar morphology to be less than two.

Figure 2 shows the relationship between the overall variability index $I_{\sigma}$ and the long-term variability index $I_{\Delta}$ for all QSO candidates and for visually identified galaxies. For extended objects, e.g. galaxies and close visual binaries, high overall variabilities $I_{\sigma}$ were measured, yet essentialy no long-term variability is indicated. This can be understood as an effect of artificial variability caused by the lower photometric accuracy for extended objects compared to stars. A significant fraction of the stars also shows only overall variability yet no long-term variability. For the identified AGNs, however, a positive correlation between $I_{\sigma}$ and $I_{\Delta}$ is clearly seen. This different behaviour supports the viewpoint (e.g., Hawkins 1983; Scholz et al. 1997; Meusinger et al. 1999) that the presence of longterm variability is an important and efficient indicator for the variability-based selection of QSO candidates. As is

${ }^{1}$ Vizier Service at the Centre de Données astronomiques des Strasbourg. 
Table 3. QSOs from the present survey. In the five columns on the right hand side, the indices defined in paper 1 for proper motion $\left(I_{\mu}\right)$, overall variability $\left(I_{\sigma}\right)$, long-term variability $\left(I_{\Delta}\right)$, and image structure $\left(I_{\text {nst }}=I_{\text {nonstellar }}\right.$ and $\left.\Psi\right)$ are given.

\begin{tabular}{|c|c|c|c|c|c|c|c|c|c|c|c|c|}
\hline no & $\alpha(\mathrm{J} 2000)$ & $\delta(\mathrm{J} 2000)$ & $z$ & $M_{B}$ & $\bar{B}$ & $\overline{U-B}$ & $\overline{B-V}$ & $I_{\mu}$ & $I_{\sigma}$ & $I_{\Delta}$ & $I_{\mathrm{nst}}$ & $\Psi / 10^{3}$ \\
\hline 1 & 171755.55 & +443228.4 & 2.625 & -28.0 & 19.0 & -0.73 & 0.24 & 1.81 & 3.13 & 3.35 & -0.84 & 0.31 \\
\hline 2 & 172333.64 & -424418.3 & 2.409 & -27.5 & 19.2 & -0.64 & 0.19 & 1.92 & 2.25 & 3.70 & -0.42 & 0.03 \\
\hline 3 & 170850.98 & -433223.7 & 2.391 & -28.7 & 18.0 & -0.56 & 0.24 & 2.19 & 3.18 & 3.36 & -1.10 & 0.69 \\
\hline 4 & 172217.25 & +424101.5 & 2.383 & -27.5 & 19.1 & -0.60 & 0.43 & 0.91 & 3.54 & 4.81 & -0.69 & 0.57 \\
\hline 5 & 171721.52 & +442120.9 & 2.363 & -27.9 & 18.7 & -0.46 & 0.37 & 1.83 & 2.91 & 1.09 & -1.21 & 1.20 \\
\hline 6 & 172154.18 & +435805.5 & 2.353 & -27.2 & 19.4 & -0.71 & 0.72 & 0.59 & 7.14 & 2.33 & 0.99 & 0.98 \\
\hline 7 & 172254.47 & +441114.4 & 2.237 & -27.8 & 18.6 & -0.68 & 0.00 & 1.63 & 2.95 & 1.83 & -1.15 & 1.14 \\
\hline 8 & 171921.14 & +413909.0 & 2.109 & -27.1 & 19.2 & -1.00 & 0.18 & 0.82 & 5.40 & 4.36 & 0.70 & 1.29 \\
\hline 9 & 171650.45 & +442452.1 & 1.976 & -27.2 & 19.0 & -1.08 & 0.49 & 1.54 & & -0.61 & -0.60 & -0.32 \\
\hline 10 & 172037.19 & 442122.7 & 1.961 & -26.6 & 19.5 & -1.30 & 0.29 & 2.21 & & 0.02 & & \\
\hline 11 & 171059.76 & 430733.8 & 1.902 & -26.5 & 19.5 & -0.81 & 0.62 & 1.48 & 1.72 & & -3.66 & 1.92 \\
\hline 12 & 171125.22 & 35208.6 & 1.833 & -26.7 & 19.1 & -1.30 & 0.07 & 1.87 & 0.03 & 2.19 & -1.45 & 0.56 \\
\hline 13 & 171634.52 & 34954.0 & 1.734 & -27.1 & 18.6 & -0.90 & 0.23 & 1.17 & 4.66 & .55 & -1.52 & 1.44 \\
\hline 14 & 171821.42 & 517.1 & 1.709 & -26.4 & 19.2 & -0.84 & 0.22 & 2.10 & 7.00 & .47 & 0.75 & 1.08 \\
\hline 15 & 171228.55 & 807.8 & 1.676 & -26.4 & 19.2 & -0.72 & 0.01 & 1.30 & & 01 & -1.28 & 1.20 \\
\hline 16 & 170903.00 & +425755.9 & 1.664 & -26.7 & 18.8 & -0.86 & -0.12 & 0.45 & & .00 & -0.89 & 0.71 \\
\hline 17 & 171330.50 & 141020.9 & 1.622 & -26.1 & 19.4 & -0.93 & 0.17 & 2.98 & 6.1 & 0.38 & -0.61 & 0.70 \\
\hline 18 & 172447.15 & +420043.6 & 1.609 & -26.8 & 18.7 & -0.60 & 0.28 & 1.24 & 6.9 & 4.34 & -0.41 & 0.15 \\
\hline 19 & 170951.08 & +435834.3 & 1.577 & -26.1 & 19.3 & -0.23 & 0.14 & 1.54 & 4.4 & 3.70 & -0.46 & 0.48 \\
\hline 20 & 171956.97 & +421310.2 & 1.531 & -25.9 & 19.4 & -0.63 & -0.52 & 0.72 & 1.83 & 1.94 & & \\
\hline 21 & 170850.73 & +423444.3 & 1.506 & -28.0 & 17.3 & -0.85 & 0.03 & 0.31 & 12.40 & 5.06 & 1.05 & 0.77 \\
\hline 22 & 171841.88 & +424337.9 & 1.473 & -26.1 & 19.2 & -0.84 & -0.10 & 2.45 & 9.30 & 3.06 & -0.05 & 1.38 \\
\hline 23 & 172224.68 & +433112.9 & 1.460 & -26.1 & 19.1 & -0.91 & 0.15 & 2.18 & & 3.99 & -1.46 & 1.13 \\
\hline 24 & 172108.57 & 432511.8 & 1.43 & -26.4 & 18.8 & -0.70 & 0.24 & 0.89 & & & -0.82 & 0.55 \\
\hline 25 & 172052.88 & 441006.8 & 1.43 & -26.1 & 19.1 & -0.69 & 0.27 & 2.60 & & & -0.66 & 0.63 \\
\hline 26 & 170931.88 & 432436.3 & 1.40 & -26.4 & 18.7 & -0.72 & & 0.86 & & & 0.19 & 0.78 \\
\hline 27 & $17 \quad 1002.12$ & 435754.3 & 1.2 & -26.1 & 18.8 & -1.03 & & 3.13 & & & -0.37 & -0.00 \\
\hline 28 & 17090 & 015.7 & 1.2 & -26.7 & 18.2 & -0.63 & & 0.6 & & & -1.12 & 0.91 \\
\hline 29 & $171^{\prime}$ & 03.6 & 1.228 & -25.5 & 19.3 & -0.71 & 0. & 0.8 & 6.2 & 1 & 1.77 & 1.16 \\
\hline 30 & 171 & 07.4 & 1.20 & -26.4 & 18.3 & -1.14 & 0.62 & 0.23 & & & -1.22 & 0.54 \\
\hline 31 & 171 & 0.0 & 1.16 & -26.2 & 18.5 & -0.86 & 0.13 & 1.91 & & & 0.04 & 1.26 \\
\hline 32 & 171 & 1.5 & 1.112 & -25.4 & 19.1 & -0.99 & 0.27 & 1.4 & & 2.0 & -0.64 & 0.16 \\
\hline 33 & 17140 & 22819.9 & 1.096 & -25.1 & 19.4 & -0.88 & 0.17 & 1.45 & 4.7 & 0.74 & 0.15 & 0.00 \\
\hline 34 & 17105 & 20012.3 & 1.089 & -25.2 & 19.3 & -0.44 & -0.04 & 0.24 & 12.8 & 2.33 & & \\
\hline 35 & 171515.32 & +422407.5 & 1.048 & -25.7 & 18.7 & -0.94 & 0.29 & 2.18 & 6.79 & 5.05 & -0.81 & -0.09 \\
\hline 36 & 171647.14 & +432753.5 & 0.978 & -24.8 & 19.4 & -0.83 & 0.17 & 1.47 & 4.3 & 3.36 & -0.17 & 0.45 \\
\hline 37 & 171109.60 & +421043.1 & 0.888 & -25.4 & 18.6 & -0.92 & 0.26 & 1.49 & 5.22 & 1.17 & -1.52 & 0.44 \\
\hline 38 & 171202.78 & +441336.9 & 0.874 & -24.8 & 19.1 & -0.98 & 0.32 & 2.13 & 12.01 & 6.65 & 1.06 & 1.53 \\
\hline 39 & 171621.16 & +415935.3 & 0.847 & -25.9 & 18.0 & -0.86 & 0.58 & 2.35 & & 8.34 & -0.27 & 0.51 \\
\hline 40 & 172136.51 & +431039.4 & 0.846 & -26.1 & 17.7 & -0.78 & 0.55 & 0.58 & 15.85 & 7.94 & -0.03 & 0.67 \\
\hline 41 & 172108.67 & +443200.3 & 0.823 & -24.9 & 19.0 & -1.09 & 0.62 & 2.15 & & 2.80 & -0.44 & 0.08 \\
\hline 42 & 170925.28 & 424013.9 & 0.799 & -24.3 & 19.4 & -0.75 & & 1.1 & & 1.61 & -0.24 & 0.15 \\
\hline 43 & 17173 & 3 & & -24.2 & 19.4 & -0 . & & 1.6 & & & & \\
\hline 44 & 1714 & 9 & & -24 & 18 & -0 & & & & & 1.23 & 0.51 \\
\hline 45 & 17200 & 018.3 & 0.7 & -24.2 & 19.3 & -0.42 & -0 . & 0. & & & & \\
\hline 46 & 17164 & 20244.8 & 0.724 & -25.0 & 18.5 & -0.75 & 0.27 & 2.3 & 8.2 & 4.7 & -0.75 & 0.96 \\
\hline 47 & 171555 & 017.4 & 0.685 & -24.8 & 18.5 & -0.73 & 0.11 & 1.1 & 23.7 & 9.1 & -0.29 & 0.44 \\
\hline 48 & 172148.26 & +435315.6 & 0.651 & -25.0 & 18.2 & -0.60 & 0.14 & 2.50 & 12.81 & 7.02 & -0.84 & 0.79 \\
\hline 49 & 172156.64 & +433947.8 & 0.649 & -25.5 & 17.7 & -0.44 & -0.02 & 1.69 & 19.76 & 8.40 & -0.52 & 0.86 \\
\hline 50 & 171730.12 & +431940.9 & 0.632 & -23.6 & 19.5 & -0.45 & & 0.75 & 1.30 & 1.95 & -2.09 & 1.39 \\
\hline 51 & 171007.61 & +431519.3 & 0.623 & -24.5 & 18.5 & -0.57 & -0.08 & 2.78 & 16.78 & 7.20 & -0.72 & 0.73 \\
\hline 52 & 171834.97 & +421125.4 & 0.525 & -23.4 & 19.2 & -0.74 & 0.33 & 2.03 & 4.31 & 4.32 & 0.25 & 0.71 \\
\hline 53 & 171951.89 & +430148.9 & 0.506 & -23.3 & 19.2 & -0.96 & 0.04 & 0.62 & 4.53 & 3.13 & -0.09 & 0.02 \\
\hline 54 & 171502.44 & +425845.0 & 0.492 & -23.3 & 19.2 & -1.07 & 0.49 & 1.54 & 3.06 & 3.23 & & \\
\hline 55 & 172358.66 & +423844.0 & 0.489 & -24.3 & 18.2 & -0.96 & 0.07 & 1.12 & 16.28 & 6.84 & -1.60 & 0.38 \\
\hline 56 & 171458.26 & +433631.0 & 0.449 & -23.0 & 19.2 & -0.71 & 0.20 & 1.06 & & 5.77 & 0.15 & 0.43 \\
\hline 57 & 170906.17 & +420157.5 & 0.30 & -23.0 & 18.4 & -1.12 & 0.53 & 1.35 & & 8.21 & 1.65 & 1.70 \\
\hline 58 & 171503.11 & +442952.1 & 0.152 & -23.1 & 16.8 & -0.86 & 0.28 & 1.97 & 26.87 & 8.57 & 6.57 & 1.04 \\
\hline
\end{tabular}


Table 4. As Table 3, but for the Seyfert 1 galaxies from the present survey.

\begin{tabular}{rllllllllllll}
\hline nо & $\alpha(\mathrm{J} 2000)$ & $\delta(\mathrm{J} 2000)$ & $z$ & $M_{B}$ & $\bar{B}$ & $\overline{U-B}$ & $\overline{B-V}$ & $I_{\mu}$ & $I_{\sigma}$ & $I_{\Delta}$ & $I_{\mathrm{nst}}$ & $\Psi$ \\
\hline 59 & 172205.70 & +424009.2 & 0.423 & -22.9 & 19.3 & -0.87 & 0.06 & 2.20 & 2.85 & 3.16 & -0.30 & 0.43 \\
60 & 172017.14 & +424001.6 & 0.357 & -22.2 & 19.6 & -0.94 & -0.50 & 0.86 & 1.29 & 1.34 & \\
61 & 171637.55 & +423912.3 & 0.246 & -21.7 & 19.2 & -0.74 & -0.11 & 1.30 & 3.02 & 1.65 & -1.01 & 1.21 \\
62 & 172105.10 & +415258.1 & 0.215 & -21.9 & 18.8 & -0.82 & 0.62 & 0.67 & 4.41 & 2.56 & 2.43 & 1.88 \\
63 & 171215.46 & +440249.5 & 0.197 & -21.8 & 18.7 & -0.99 & 0.51 & 3.62 & 16.60 & 6.04 & 1.85 & 0.89 \\
64 & 171302.56 & +431722.2 & 0.178 & -21.0 & 19.2 & -0.61 & 0.58 & 0.68 & 10.40 & 2.64 & & \\
65 & 172201.99 & +431524.0 & 0.137 & -21.7 & 18.0 & -0.89 & 0.97 & 2.08 & 117.70 & 10.84 & 0.42 & 1.08 \\
\hline
\end{tabular}

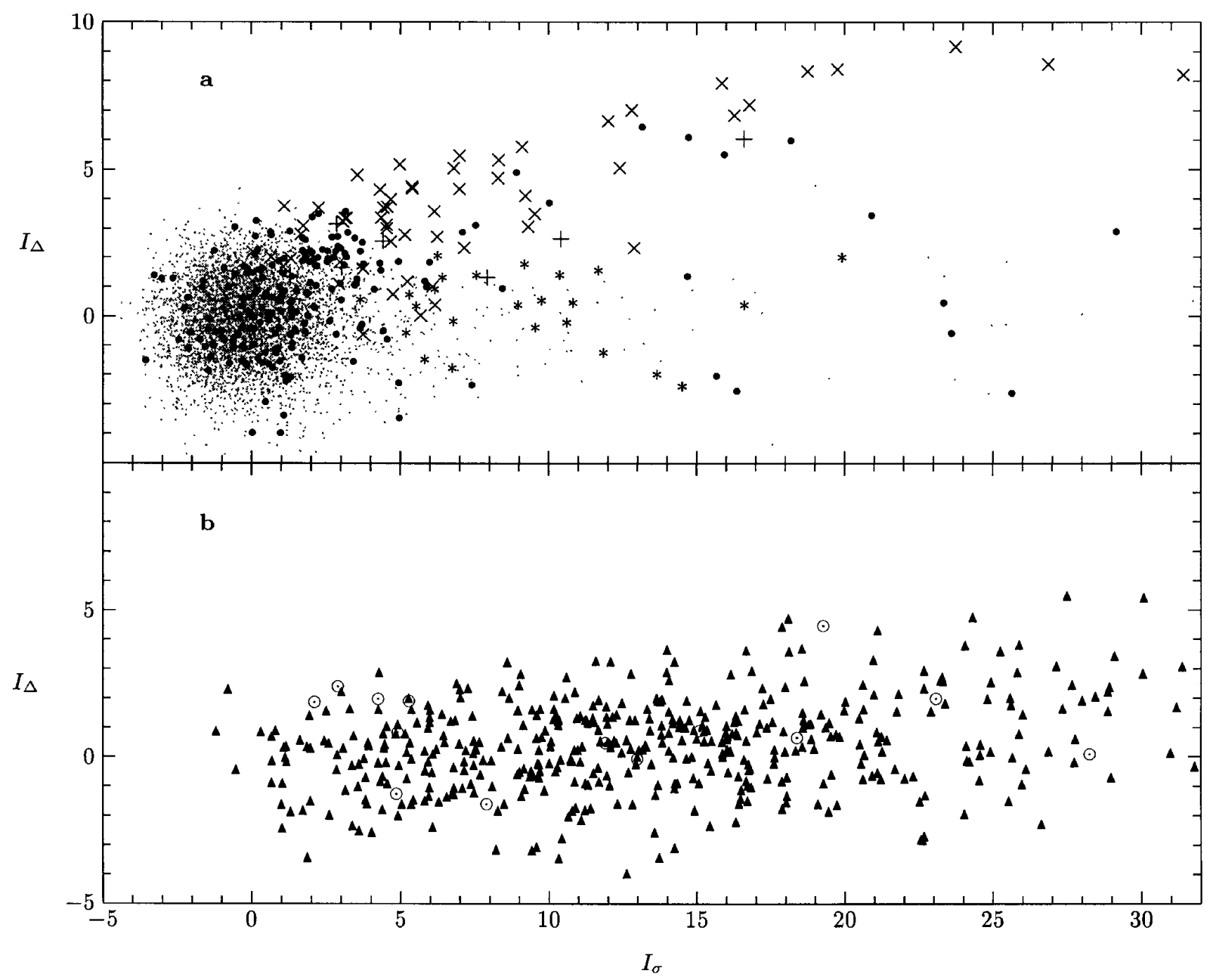

Fig. 2. Long-term variability index $I_{\Delta}$ versus overall variability index $I_{\sigma}$, a) for all QSO candidates with $B \geq 16.5$ (gray dots), established QSOs $(\times)$, Seyferts $(+)$, NELGs $(*)$ and spectroscopically identified stars $(\bullet)$. For comparison, visually identified galaxies $(\mathbf{\Lambda})$ and visual double stars $(\odot)$ are shown in panel b). Note that the object No. 65 is, with $I_{\sigma}=118$ and $I_{\Delta}=10.84$ outside this diagram.

obvious from Fig. 2, an AGN-candidate sample constructed of star-like, stationary objects with both high $I_{\sigma}$ and high $I_{\Delta}$ has a low contamination.

Low-luminosity AGNs (LLAGNs) have probably shorter variability timescales than QSOs (Filippenko 1992; Lira et al. 1999; Moran et al. 1999). Samples of slightly extended objects having large overall variability indices, yet small long-term variability indices may include such
LLAGNs. Although our survey does not primarily aim at such objects, we found a surprisingly large number of NELGs (Meusinger \& Brunzendorf 2000), which appear nearly star-like on Schmidt plates, but are recognized as galaxies on the CAFOS acquisition frames taken under $1^{\prime \prime}$-seeing conditions. The quality of the follow-up spectra is not sufficient to discriminate between AGNs and H II galaxies by means of diagnostic line ratios. In a 
Table 5. Equivalent widths $E W$ of the prominent emission lines in the composite spectrum (Fig. 3) as well as the corresponding full widths at half maximum $F W H M$. The measured $F W H M$ s of the [O III] lines are due to the limited linear resolution of the observations.

\begin{tabular}{lrrr}
\hline line & $\begin{array}{r}E W \\
(\AA)\end{array}$ & $\begin{array}{r}F W H M \\
(\AA)\end{array}$ & $\begin{array}{r}F W H M \\
\left(\mathrm{~km} \mathrm{~s}^{-1}\right)\end{array}$ \\
\hline $\mathrm{Ly} \alpha+\mathrm{N}$ v $\lambda 1240$ & $55 \pm 5$ & $13 \pm 1$ & $3200 \pm 300$ \\
Si IV+O IV] $\lambda 1400$ & $5 \pm 1$ & $23 \pm 1$ & $4900 \pm 300$ \\
$\mathrm{C}$ IV $\lambda$ 1549 & $41 \pm 2$ & $27 \pm 1$ & $5200 \pm 200$ \\
$\mathrm{C}$ III $]$ 1909 & $22 \pm 2$ & $40 \pm 1$ & $6300 \pm 200$ \\
$\mathrm{Mg}$ II $\lambda 2798$ & $40 \pm 2$ & $36 \pm 1$ & $3900 \pm 100$ \\
$\mathrm{H} \gamma$ & $25 \pm 3$ & $50 \pm 1$ & $3500 \pm 100$ \\
$\mathrm{H} \beta$ & $65 \pm 6$ & $44 \pm 2$ & $2700 \pm 200$ \\
{$[\mathrm{O}$ III] $\lambda 4959$} & $10 \pm 2$ & $20 \pm 1$ & $1200 \pm 100$ \\
{$[\mathrm{O}$ III] $\lambda 5007$} & $34 \pm 2$ & $20 \pm 1$ & $1200 \pm 100$ \\
$\mathrm{H} \alpha+[\mathrm{N}$ II $] \lambda 6584$ & $200 \pm 10$ & $35 \pm 2$ & $1600 \pm 100$ \\
\hline
\end{tabular}

separate observation campaign, we have obtained spectra of higher resolution and higher signal-to-noise ratio for most NELGs. The discussion of the spectra and other properties of the NELGs will be the aim of a separate study.

\section{Sample properties of the VPM QSOs}

\subsection{Emission lines}

The spectra exhibited in Fig. 9 are dominated by the typical QSO emission lines: Ly $\alpha \lambda 1216$, Si IV+O IV] $\lambda 1400$, C IV $\lambda 1549, \mathrm{C}$ III] $\lambda 1909, \mathrm{Mg}$ II $\lambda 2798$, the Balmer series, and the [O III] $\lambda 4959$ and $\lambda 5007$ lines. There are no QSOs in Fig. 9 that obviously show peculiar spectra. We only note that the QSO No. 3, which is the most luminous of the survey, seems to have strong absorption features. As is typical for QSOs with broad absorption lines (Turnshek 1988), this object has a comparatively weak CIV emission and has no radio-counterpart in the FIRST catalogue (White et al. 1997), i.e. it is not an intense radio source. Various explanations for QSOs with weak emission lines are discussed by McDowell et al. (1995). With regard to the low spectral resolution and the relatively low signalto-noise ratio, we omit a more detailed discussion of the individual spectra from Fig. 9.

In Fig. 3, we show the composite spectrum obtained from the superposition of the individual spectra from all 65 QSOs and Seyfert 1s after an approximate fluxcalibration and transformation/rebinning of the wavelength scale into the rest frame. The flux-calibrated spectra have been co-added weighting each spectrum according to its local signal-to-noise ratio measured in the continuum. The resulting composite spectrum is exceptionally similar to the ones presented by Cristiani \& Vio (1990) and Francis et al. (1991). Besides the major emission lines mentioned above, several weaker features are recognized as well, e. g. He II $\lambda 1640, \mathrm{~N}$ III] $\lambda 1750$, [Ne V] $\lambda 3426$, [O II] $\lambda 3727$, [Ne III] $\lambda 3869$ and $\lambda 3969$. Broad emission fea- tures which are interpreted as blended Fe II resonance lines are also visible in Fig. 3.

Sample-averaged line equivalent widths $(E W \mathrm{~s})$ have been measured from the composite spectrum in Fig. 3; note that these are line widths in the rest frame and are independent on wether the spectra are flux-calibrated or not. The results are listed in Table 5. The comparison with reference samples of radio-quiet QSOs (e.g., Cristiani \& Vio 1990; Francis et al. 1991; Green 1996; Zheng et al. 1997; Puchnarewicz et al. 1997) yields a good agreement. On the other hand, our $E W$ values are lower than those measured for pure samples of Seyfert 1 galaxies (Véron-Cetty et al. 1983), reflecting the known luminosity dependence (Baldwin-effect; Baldwin 1977; Baldwin et al. 1989). For the AGNs from the present sample, a significant anti-correlation between luminosity and $E W$ is found only for C III].

\subsection{Broad-band colours}

Since we abandoned an accurate flux calibration, the follow-up spectra contain only limited information about the continuum radiation. Thus, a direct comparison of the continua of VPM QSOs with those of QSOs from other surveys seems not possible. However, the SED of the continuum is also measured by the broad-band colour indices. In the previous subsection, we have shown that the $E W \mathrm{~s}$ of the pronounced emission lines in VPM QSOs do not differ from those of QSO in other samples. Hence, differences in the colour indices, if significant, can be attributed directly to differences in the continua.

A comparison of the colours seems to be hampered by the fact that we estimated time-averaged colours while for most comparison samples colours are derived from single observation epochs. Note, however, that we derived the colour indices from the plates taken in the years 1966 to 1973 only, with a strong concentration towards the mean epoch 1968.25 \pm 0.5 . Hence, the main effect of the averaging is the reduction of the photometric uncertainties and of the short-term variability. The presence of a long-term variability does not significantly alter the indices.

Figure 4 shows the distribution of the different object types in the $U-B$ versus $B-V$ plane. (The visually identified galaxies are not marked for the sake of clarity.) For comparison, classical selection criteria for the UVexcess search and for the two-colour search are shown as well. It is obvious from Fig. 4 that the established QSOs from the VPM survey populate the same region of the colour-colour diagram as colour-selected QSOs. On the other hand, the NELGs show a broader distribution, but this may be assigned simply to the contribution of the host galaxies, if NELGs harbour AGNs at all. All of the high-priority QSO candidates with unusually red colours (Paper I) proved to be foreground stars. The colour distribution of our variability-selected QSO sample and of colour-selected QSO samples are very similar. No unuasually red QSOs were found. Of course, we can not rule 


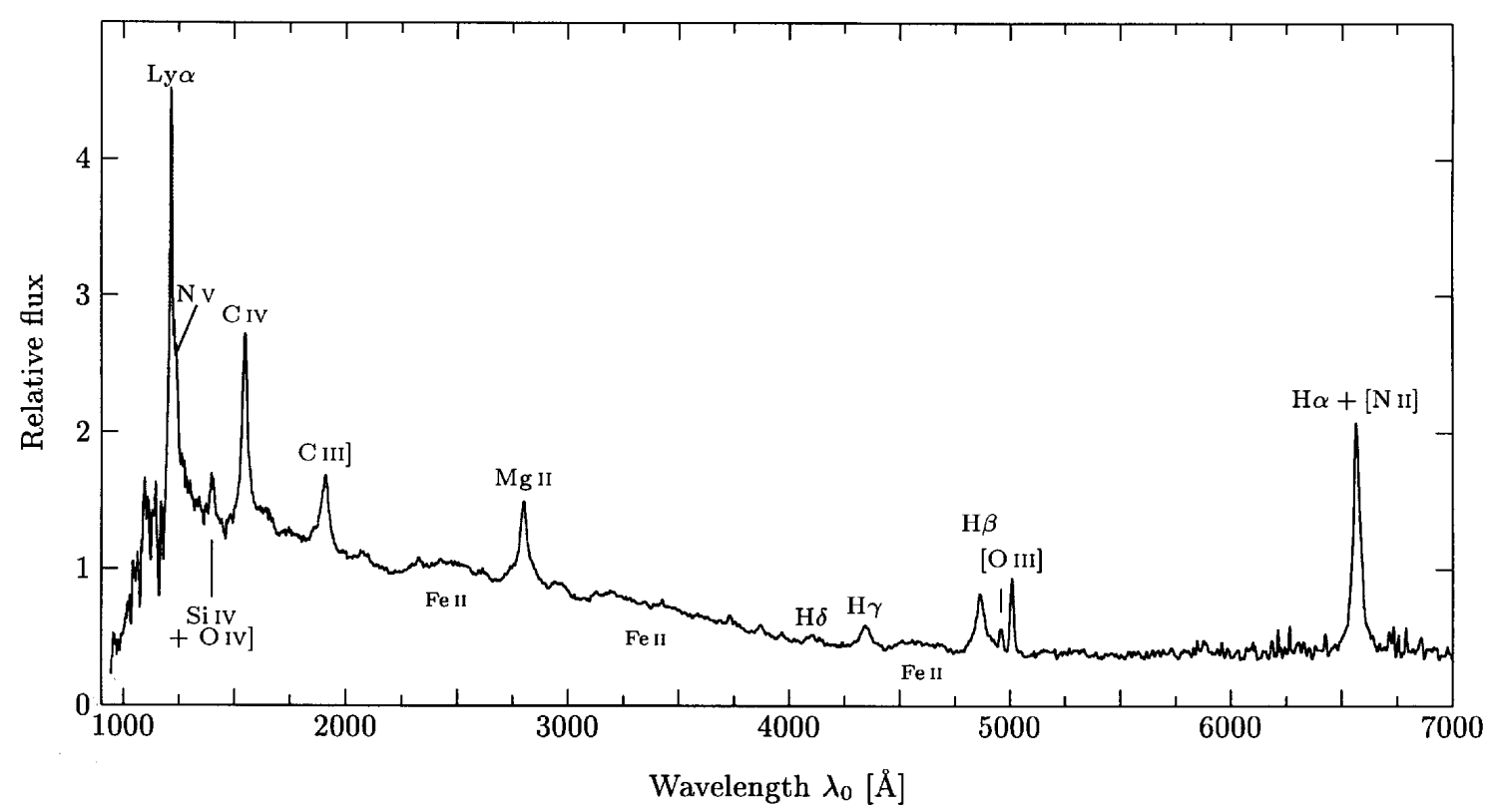

Fig. 3. Composite spectrum (in the rest frame) obtained from the low-resolution spectra for all QSOs and Seyfert 1 s from Fig. 9.

out that deeper variability-selected QSO samples may also catch a substantial number of red QSOs. An upper limit of the fraction of red QSOs up to the survey limit $\bar{B}=19.7$ is derived in Sect. 4.3.

The conclusion that VPM QSOs have a similar colour distribution as colour-selected QSOs is also illustrated by the colour-redshift plots. The observed QSO colours are determined by the shape of the continuum, the contribution of the most pronounced emission lines in the corresponding filter band-passes, and the redshift. In Fig. 5, the $z$-dependences of $U-B$ and $B-V$ are shown for the AGNs from Tables 3 and 4 in comparison with the QSOs from the catalogue by Véron-Cetty \& Véron (1998). There are no statistically significant differences between these both samples.

\subsection{Red QSOs}

It is generally believed that QSOs are blue. However, detections at radio-wavelengths (e.g., Smith \& Spinrad 1980; Rieke et al. 1982; Ledden \& O'Dell 1983; Webster et al. 1995; Stickel et al. 1996; Najita et al. 2000; Brotherton et al. 2001), as well as in the near infrared (Beichman et al. 1998), and in the X-ray band (e.g., Kim \& Elvis 1999; Vignali et al. 2000; Nakanishi et al. 2000) revealed the existence of a substantial population of QSOs with unexpected red colours. On the basis of a complete sample of radio sources from the Parkes Half-Jansky FlatSpectrum survey, Webster and co-workers (Webster et al. 1995; Drinkwater et al. 1997; Masci et al. 1999; Francis et al. 2000) found a strong population of red sources, some having extremely red $B-K$ colours. Webster et al. (1995) argued that up to $80 \%$ of all QSOs are missed by conventional UVX surveys. Although it seems now well established that many flat-radio-spectrum sources are ex- tremely red, the origin of the spread in the $B-K$ colour as well as the fraction of red QSOs overlooked by classical colour surveys remains a matter of debate (e.g., Srianand \& Kembhavi 1997; Benn et al. 1998; Masci et al.1998; Francis et al. 2000).

Our VPM survey does not explicitely use colours as search criteria and is therefore, in this respect, expected to be unbiased against red QSOs. On the other hand, the objects were selected in the $B$ band. This provides, of course, a bias against objects with a SED peaked in the red spectral range if they are faint in $B$. For instance, if the SED of the red QSOs is due to interstellar reddening, strongly reddened QSOs will tend to drop below the brightness threshold of our search. However, we would expect to find at least some of them, namely those which are sufficiently bright in $B$ (see e.g., Beichman et al. 1998; Kim \& Elvis 1999).

We found indeed a substantial fraction of high- and medium-priority candidates with unconventionally red $U-B$ and $B-V$ colours (Paper I). However, as described in Sect.4.2, all of these extraordinary candidates were spectroscopically confirmed as foreground stars, not as QSOs. An upper limit of the total fraction $f_{\text {red }}$ of red QSOs brighter than $B_{\lim }=19.7$, at a chosen significance level $\alpha=0.95$, can be derived by simple probability calculations. Under the assumption that red QSOs have similar variability properties as ordinary QSOs, the fraction $f_{\text {red }}$ calculates as

$1-\left(1-f_{\mathrm{red}}\right)^{n} \leq \alpha \quad \rightarrow \quad f_{\mathrm{red}} \leq 1-\sqrt[n]{1-\alpha}$

with $n=58$ VPM QSOs, i.e., the fraction of QSOs with unusually red optical colours in the survey field and magnitude range $B \leq 19.7$ is $f_{\text {red }} \leq 5 \%$.

We have cross-correlated our QSO/Seyfert 1 sample against the Point Source Catalogue (March 2000 edition) 


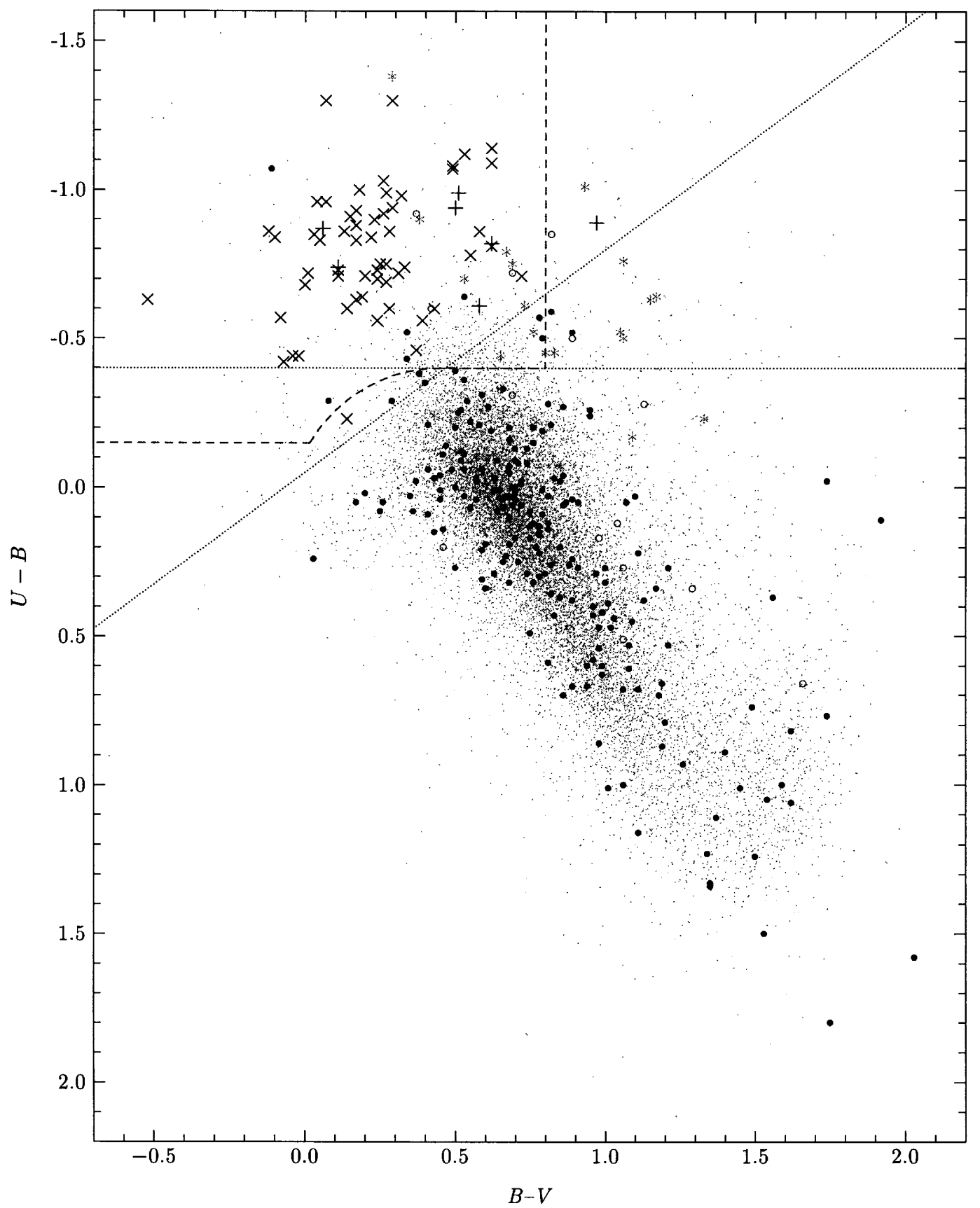

Fig. 4. Colour-colour diagram of the QSOs (crosses), Seyfert 1s (plus signs), and NELGs (asterisks) from the present study. In addition, spectroscopically identified stars (bullets), visual binaries (open circles) are shown as well as the non-identified objects with $B \leq 19$ (small dots). Selection criteria from colour surveys are indicated. Horizontal dotted line: UVX search, diagonal dotted line: two-colour search as discussed by Scholz et al. (1997), dashed curve: two-colour selection according to LaFranca et al. (1992).

from the 2 Micron All Sky Survey (2MASS; Skrutskie et al. 1997). For a search redius of $10^{\prime \prime}$, three QSOs and one Seyfert 1 were identified with 2MASS-sources. Their near-infrared magnitudes are listed in Table 6 along with the $B-K_{\mathrm{s}}$ colour index. Only one of the four objects (number 57) is also identified with a radio source. Compared with the extreme red colours of radio-loud QSOs found by Webster et al., the colour indices listed in Table 6 are rather characteristic for optically detected
QSOs. For point sources, the magnitude limit of 2MASS at a signal-to-noise ratio of 10 is given as $K_{\mathrm{s}, \lim }=14.3$. The non-detection of 2MASS counterparts for the other AGNs from our sample obviously means that there is no QSO in our sample with $B-K_{\mathrm{s}}>5.4$. For the three QSOs from Table 6 , the average $\overline{B-K} \approx 3$ corresponds approximately to an optical continuum slope of $f_{\nu} \propto \nu^{-0.3}$ predicted by the free-free continuum radiation model (Barvainis 1993). 

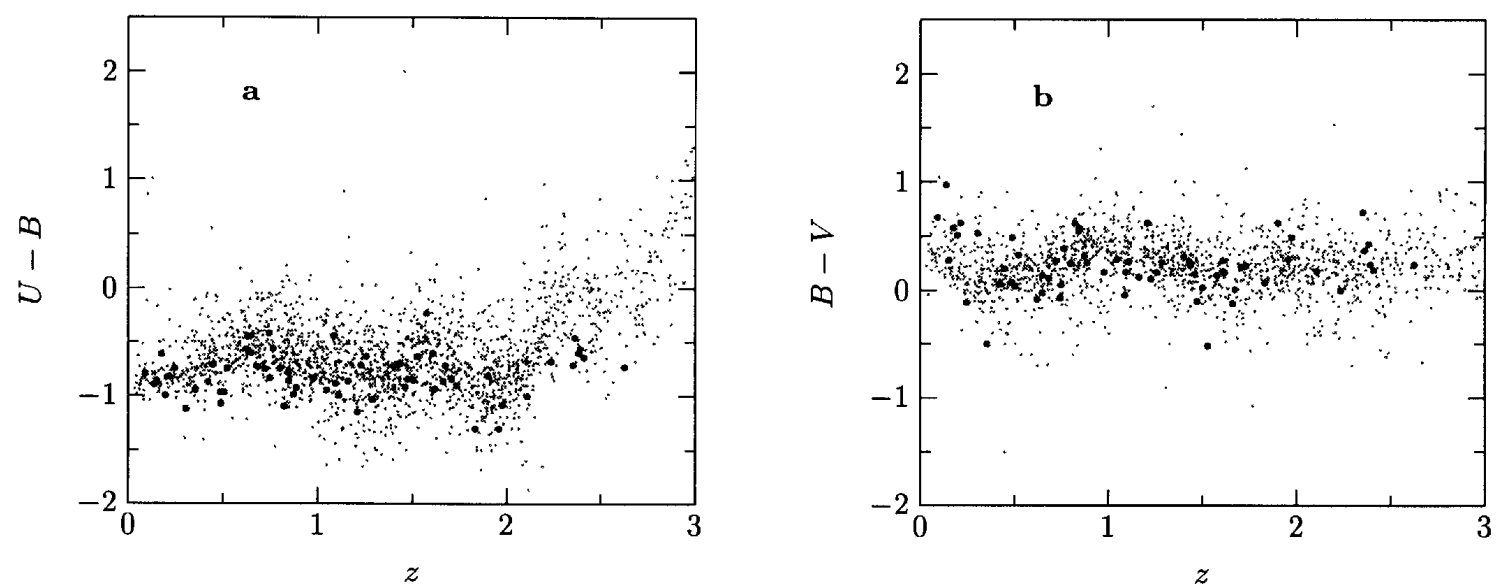

Fig. 5. Colour indices $U-B$ a) and $B-V$ b) of the identified QSO/Seyfert $1 \mathrm{~s}(\bullet)$ as a function of the redshift $z$. For the sake of comparison, the QSOs from Véron-Cetty \& Véron (1998) are plotted (grey dots).

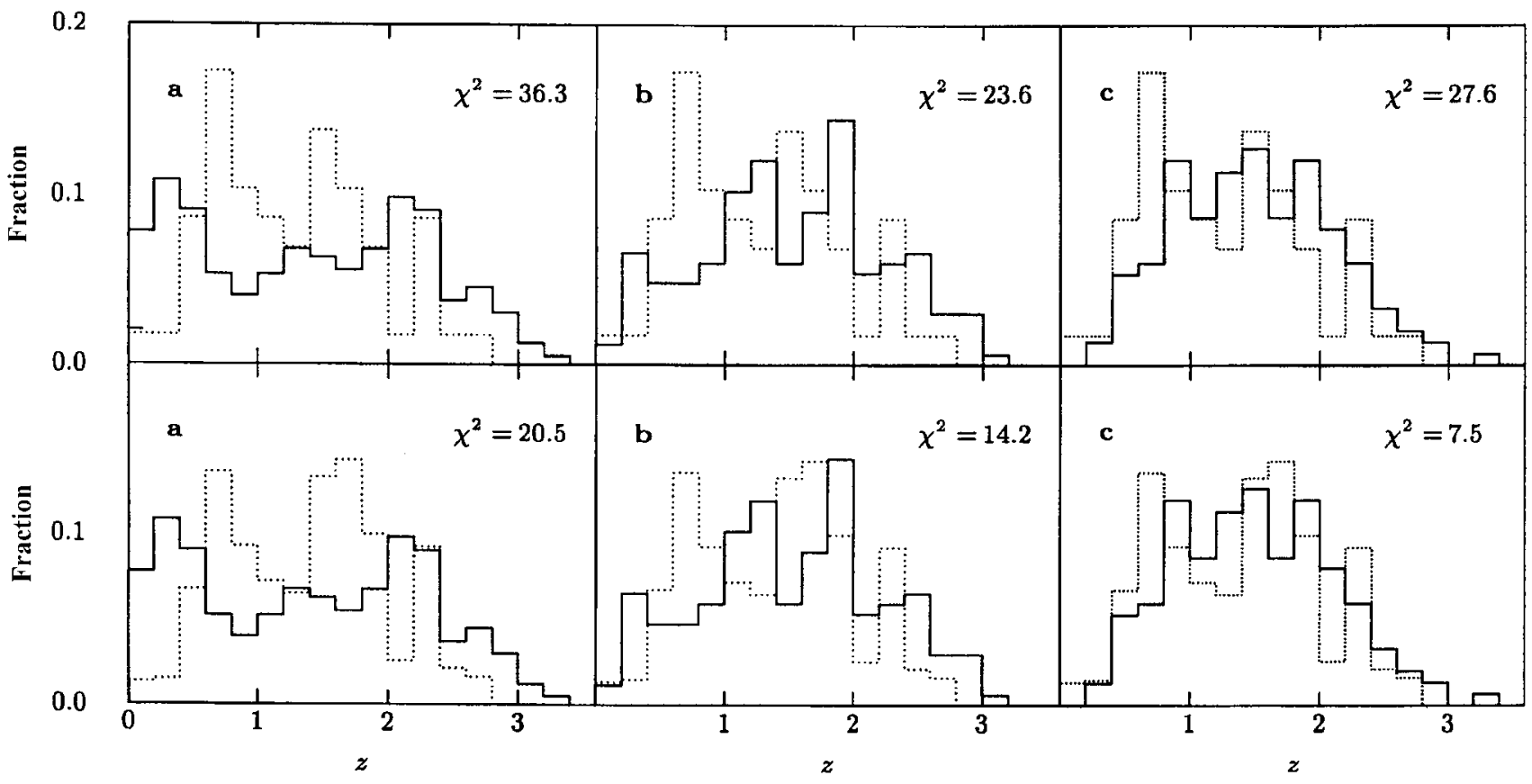

Fig. 6. Fraction of QSOs per redshift interval from the present survey (dotted histogram), compared with the normalized redshift distributions from other surveys (solid histograms): a) Hamburg Quasar Survey (Engels et al. 1998; Hagen et al. 1999), b) CFHT Blue Grens Survey (Crampton et al. 1989), c) the sample of optically selected QSOs (mainly UVX and objective prism) discussed by Cristiani et al. (1997). Top line: uncorrected VPM data; bottom line: after completeness correction of the VPM data.

Table 6. Near-infrared magnitudes and $B-K_{\mathrm{s}}$ colour index of the VPM-QSOs/Seyfert1s identified with 2MASS sources.

\begin{tabular}{ccccc}
\hline no & $J$ & $H$ & $K_{\mathrm{s}}$ & $B-K_{\mathrm{s}}$ \\
\hline 21 & $15.84 \pm 0.08$ & $15.03 \pm 0.08$ & $14.95 \pm 0.16$ & 2.3 \\
39 & $16.14 \pm 0.09$ & $15.84 \pm 0.15$ & $15.02 \pm 0.13$ & 3.0 \\
57 & $16.41 \pm 0.12$ & $15.55 \pm 0.13$ & $14.71 \pm 0.14$ & 3.7 \\
61 & $16.39 \pm 0.13$ & $15.95 \pm 0.19$ & $14.70 \pm 0.12$ & 4.1 \\
\hline
\end{tabular}

\subsection{Redshift distribution}

In Fig. 6, the $z$ distribution of the VPM QSOs is compared with published $z$ distributions from three other QSO surveys with comparable limiting magnitudes: (a) the Hamburg Quasar Survey based on objective prism plates (Engels et al. 1998; Hagen et al. 1999), (b) the CFHT Blue Grens Survey (Crampton et al. 1989), and (c) a sample of QSOs selected by the objective prism and UVX method (Cristiani et al. 1997). At a first glance, the VPM QSO redshift distribution appears stronger weighted towards lower $z$ than the distributions from the comparison samples (b) and (c) and seems different from the HQS at either side of the $z$ range. We performed a $\chi^{2}$-test where we considered the VPM data as the observed distribution and the comparison samples as the expected distributions. The binning from Fig. 6 was used, with the exceptions that the 


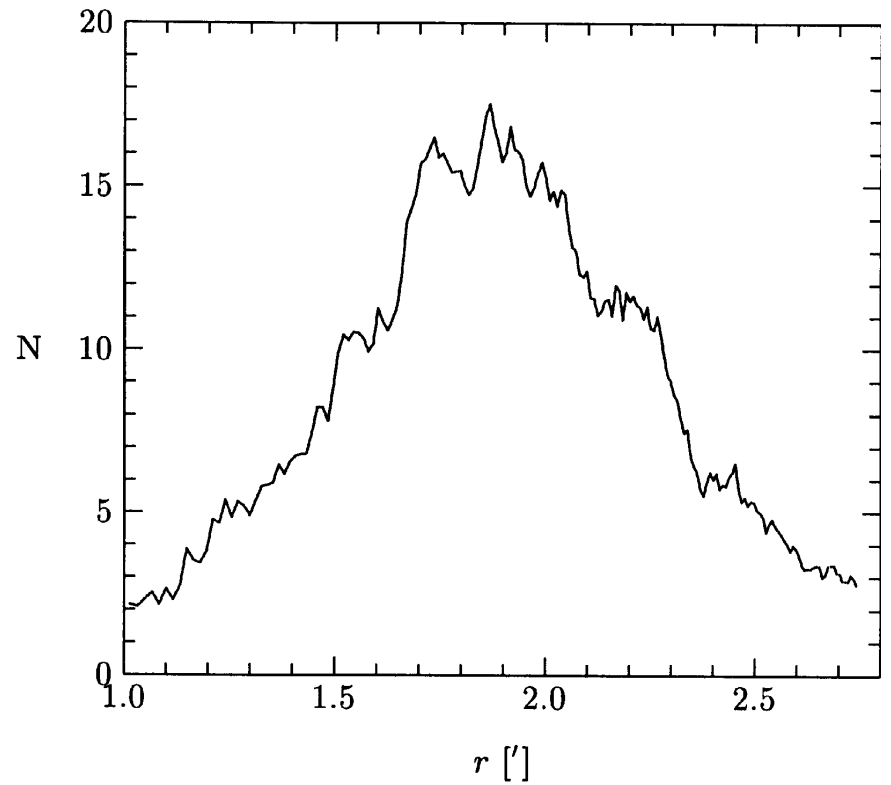

Fig. 7. Number of resolved stars per $\square^{\prime}$ as a function of the distance $r$ (in arc min) from the cluster centre of M 92 .

first bin contained all objects with $z<0.4$, and the last one all objects with $z>2.6$, respectively. The $\chi^{2}$-values for the comparison of two distributions are given in the corresponding panels of Fig. 6 . With $\chi_{0.95}^{2}=21.0$ for an error probability of 0.05 along with a number of $\nu=12$ degrees of freedom we find $\chi^{2}>\chi_{0.95}^{2}$ in all three cases, i.e. the redshift distribution of the VPM QSOs is significantly different from all three reference samples.

A bias towards lower $z$ has been predicted in Paper I as a result of the magnitude-dependent variability selection threshold and the positive correlation between the apparent magnitude and the mean redshift of QSOs. Expected incompleteness rates as a function of $z$ (Fig. 22 in Paper I) were derived by the comparison of the variability data from Hook et al. (1994) with the $B$-dependent completeness of the variability selection in the present survey. These rates can be used to correct the VPM $z$ distribution from Fig. 6. The results are shown in the bottom line of Fig. 6. The comparison of the corrected distribution yields a better agreement as compared with the uncorrected data. According to the $\chi^{2}$-test, the hypothesis that the VPM sample represents the same QSO population as the three comparison samples does not have to be rejected any more.

\subsection{Surface density}

The QSO surface density is an important characteric of a survey. In principle, it is estimated simply by dividing the number of QSOs brighter than a given magnitude by the effective search area. In the present study, the apparent magnitudes are corrected for an interstellar extinction of $A_{B}=0.08 \mathrm{mag}$.

The field size covered by the Schmidt plates is $3.3 \times 3.3$. After subtracting the areas covered by the calibration

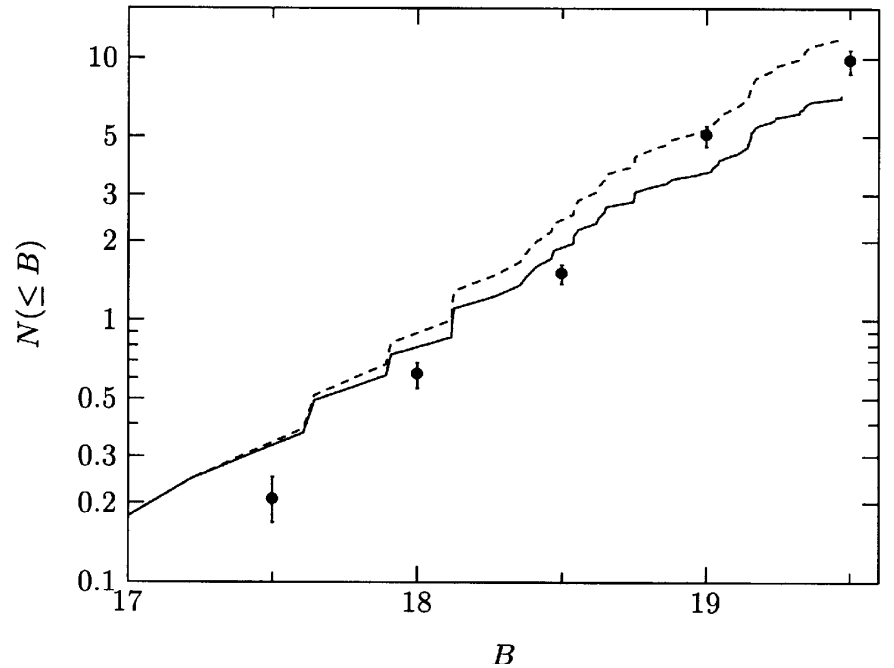

Fig. 8. Integral QSO surface density $N(\leq B)$, i. e. number of QSOs brighter than a given magnitude $B$ per square degree where $B$ has been corrected for an interstellar extinction of $A_{B}=0.08 \mathrm{mag}$. Solid polygon: VPM QSOs from Table 3, dashed polygon: incompleteness-corrected VPM data, dots with error bars: integral surface densities from Hartwick \& Schade (1990).

wedge, the plate margins, as well as the inner (crowded) region of the globular cluster M92, the effective search area is reduced to $8.58 \square^{\circ}$ (see Paper I). This value has to be corrected further by the surface area on the plates, $A_{*, \text { tot }}=N_{*} \bar{A}_{*}$, which is covered by the images of $N_{*} \approx$ 28500 objects in the remaining field. The average area, $\bar{A}_{*}$, covered by the image of a single object has been estimated in the following way: the radial profile of the number surface density of objects in M 92 (Fig. 7) shows a characteristic profile with two parts: (a) an apparently low density in the very centre (due to strong image crowding) and a strong rise of the number density as the crowding weakens with increasing centre distance, and (b) a decrease in the outer part due to radial density profile of the cluster. The maximum between these two parts represents the maximum resolvable number density $\mu_{*, \max }={\overline{A_{*}}}^{-1}=17 / \square^{\prime}$, i. e. $\bar{A}_{*}=212 \square^{\prime \prime}$ and $A_{* \text {,tot }}=0.47 \square^{\circ}$. This means that a QSO is not detected as an individual, separate object if it has a nearby neighbour within a distance of, on the average, about $8^{\prime \prime}$. Hence, the effective survey area amounts to $8.11 \square^{\circ}$.

The resulting integral (cumulative) surface density is shown in Fig. 8 compared with the QSO surface density derived by Hartwick \& Schade (1990) from the combination of different optical survey data. For colour-selected samples it became customary to distinguish between low redshifts $(z<2.2)$ and medium redshifts $(2.2 \leq z<3)$. We do not make such a subdivision as our sample is too small and as such a division is motivated by the selection effects of the search techniques rather than by physical arguments. As discussed already in Paper I, the surface densities based on single-epoch observations are affected by variability and cannot be compared directly with the 


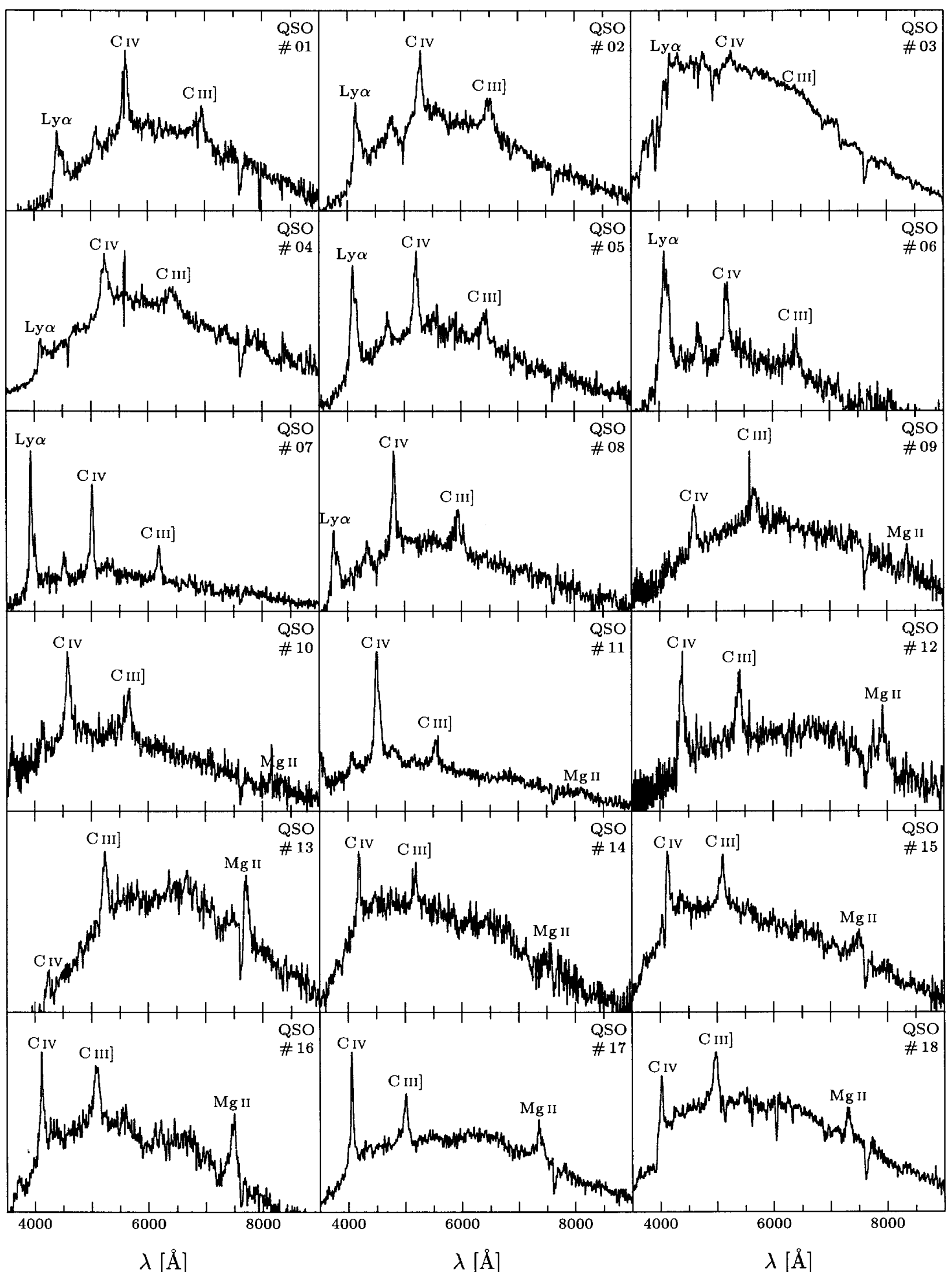

Fig. 9. Low-resolution spectra (uncalibrated flux versus wavelength) of all QSOs and Seyfert 1s from the present study. In the upper right corner of each panel, the object type and the running number from Tables 3 and 4, respectively, are given. 


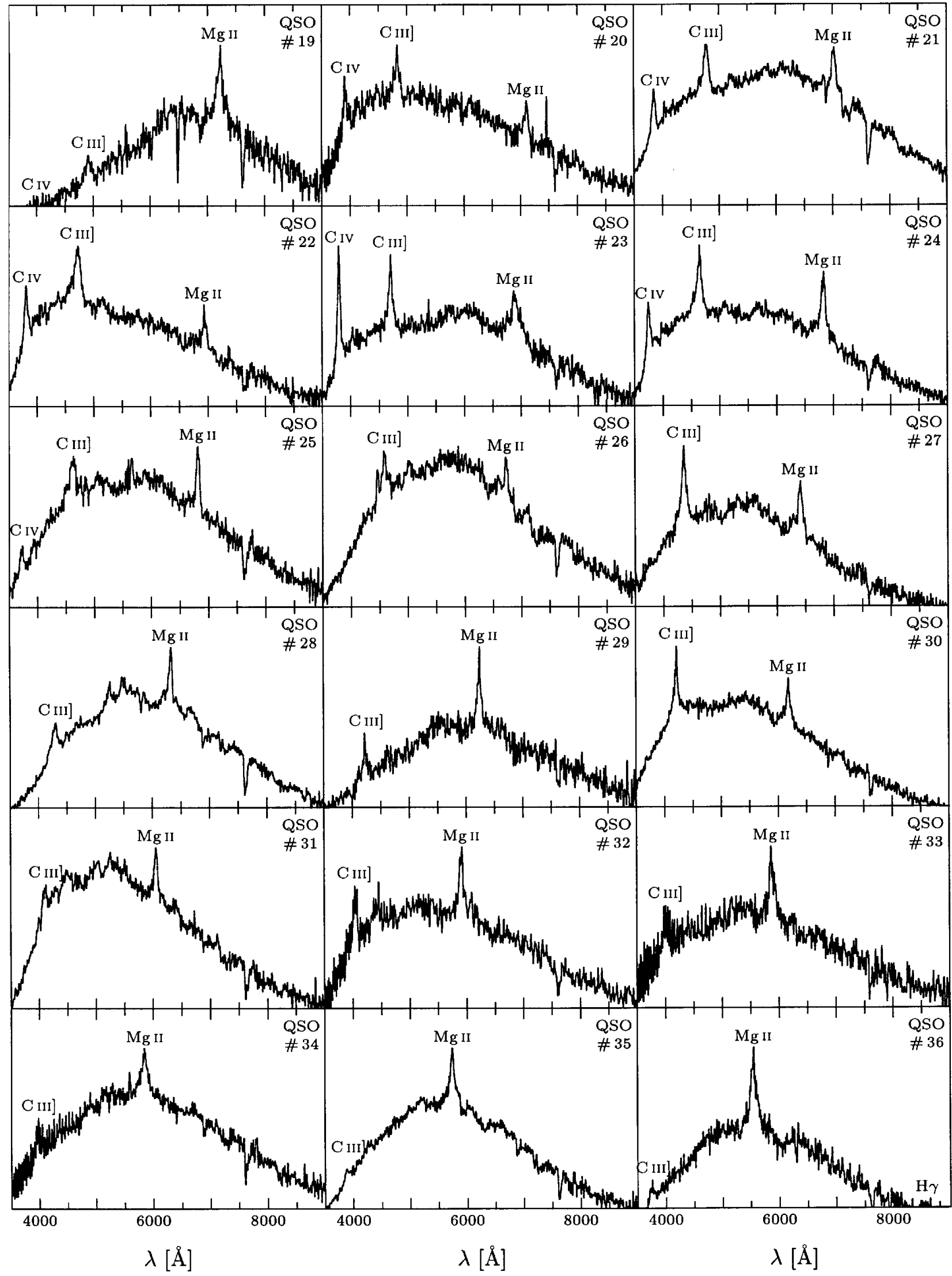

Fig. 9. continued. 
H. Meusinger and J. Brunzendorf: VPM QSO survey in the M 92 field. II.

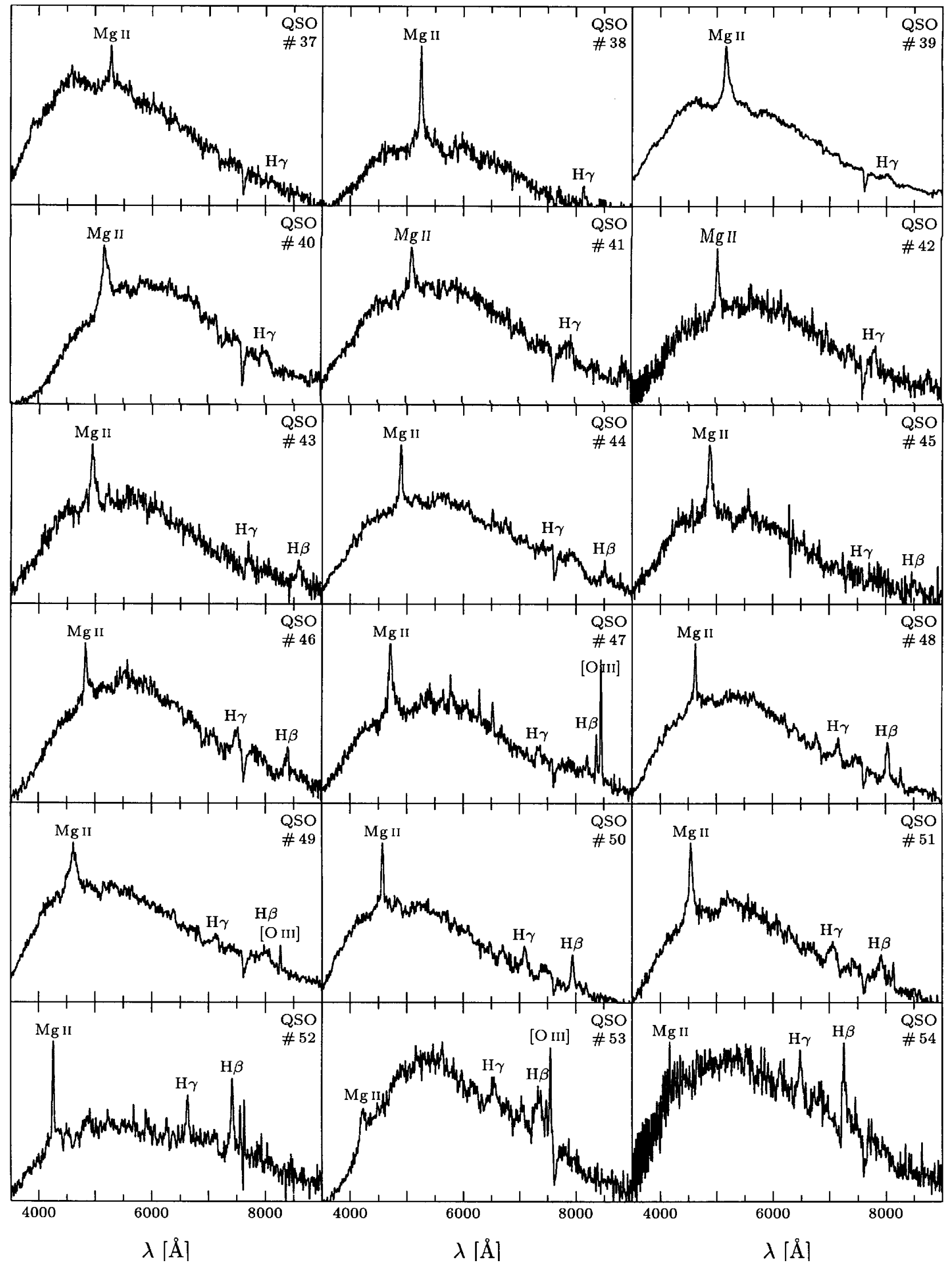

Fig. 9. continued. 

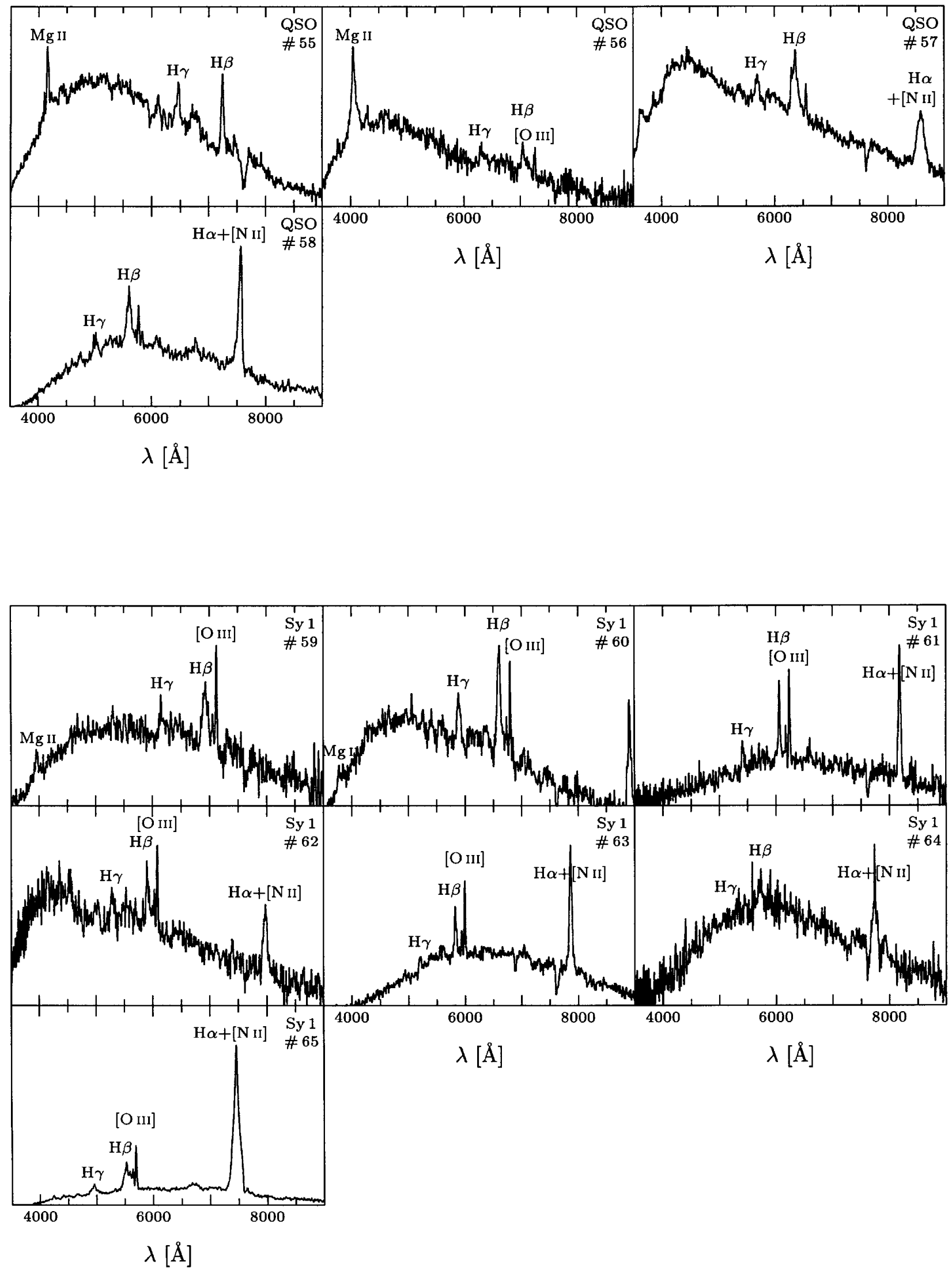

Fig. 9. continued. 
surface densities based on time-averaged magnitudes. Note, however, that the data given by Hartwick \& Schade were corrected for such a variability-induced overcompleteness. Figure 8 indicates an overabundance of bright $(B \lesssim 18)$ VPM QSOs and an underabundance of faint ones, as compared with the data by Hartwick \& Schade. An underabundance of VPM QSOs with faint apparent magnitudes is expected simply as a consequence of the magnitude-dependent incompleteness discussed at length in Paper I. If we take into account the incompleteness factors estimated in Paper I, the corrected surface densities are in a perfect agreement with the data from Hartwick \& Schade at $B \approx 19$. On the other hand, the relative overabundance of apparently bright VPM QSOs is an interesting result, but has to be taken with care due to the limitations of small-number statistics: there are only 5 VPM QSOs with corrected $B$ magnitudes less than 18. (In the context of the luminosity function of QSOs, Hartwick \& Schade adopt $q_{0}=0.5$ which is different from $q_{0}=0$ in the present paper. It should be noticed that this difference has no significant influence on the comparison of the surface density-magnitude relation discussed above.)

\section{Conclusions}

We performed a VPM QSO search in a 10 square degrees field on 162 selected digitised $B$ Schmidt plates with a time-baseline of more than three decades. We have demonstrated that this method provides an efficient search strategy for QSOs. As a result of spectroscopic follow-up observations of the candidates with $B \leq 19.7,65$ QSOs and Seyfert 1 galaxies and 27 narrow-emission line galaxies were identified with redshifts $z \approx 0$ to 3 and absolute magnitudes $-21 \lesssim M_{B} \lesssim-29$. The most important conclusion from the present study is that there is no significant difference between the properties of the QSOs from the VPM sample and those from more traditional methods. In particular, both the optical broad-band colour indices and the spectral features do not provide a hint on a substantial population of red QSOs up to the limit of the present survey, provided that their variability properties are not different from those of the non-red QSOs. As a consequence, we suggest that the selection strategy of the VPM survey can be combined with colour search criteria without a significant loss of completeness of the QSO search, at least in the magnitude range $B \lesssim 19.5$. Such a combined VPM-colour survey is expected to reach a very high search efficiency.

Acknowledgements. This paper formed part of J. B.'s Ph.D. Thesis. J. B. acknowledges financial support from the Deutsche Forschungsgemeinschaft under grants Me1359/3 and Me1350/8. The staff of the DSAZ at Calar Alto, Spain, is grately acknowledged for their support during the observation campaigns. We gratefully acknowledge M. Ball for his support during the observations with TAUMOK. We would also like to thank the referee, Dr. M. Véron-Cetty for her useful comments that led to the improvement of our work.

This reasearch has made use of the VizieR service which is operated at CDS, Strasbourg, France, and of the NASA/IPAC Extragalactic Database (NED) which is operated by the Jet Propulsion Laboratory, California Institute of Technology, under contract with the National Aeronautics and Space Administration.

\section{References}

Baldwin, J. A. 1977, ApJ, 214, 679

Baldwin, J. A., Wampler, E. J., \& Gaskell, C. M. 1989, ApJ, 338,630

Ball, M. 2000, Ph.D. Thesis, University Jena

Barvainis, R. 1993, ApJ, 412, 513

Benn, C. R., Vigotti, M., Carballo, R., Gonzalez-Serrano, J. I., \& Sánchez, S. F. 1998, MNRAS, 295, 451

Bershady, M. A., Trevese, D., \& Kron, R. G. 1998, ApJ, 496, 103

Beichman, C. A., Chester, T. J., Cutri, R., Lonsdale, C. J., \& Kirkpatrick, D. 1998, PASP, 110, 367

Brotherton, M. S., Arav, N., \& Becker, R. H., et al. 2001, ApJ, 546,134

Brunzendorf, J., \& Meusinger, H. 1999, A\&AS, 139, 141

Brunzendorf, J., \& Meusinger, H. 2000, Astron. Ges. Abstr. Ser., 17,87

Brunzendorf, J., \& Meusinger, H. 2001, A\&A, 373, 38, Paper I

Crampton, D., Cowley, A. P., \& Hartwick, F. 1989, ApJ, 345, 59

Cristiani, S., \& Vio, R. 1990, A\&A, 227, 385

Cristiani, S., Trentini, S., LaFranca, F., \& Andreani, P. 1997, A\&A, 321, 123

Drinkwater, M. J., Webster, R. L., Francis, P. J., et al. 1997, MNRAS, 284, 85

Engels, D., Hagen, H.-J., \& Cordis, L., et al. 1998, A\&AS, 128, 507

Filippenko, A. V. 1992, in ASP Conf. Ser. 31, Relationships between active galactic nuclei and starburst galaxies, ed. A. V. Filippenko, 253

Francis, P. J., Hewett, C., \& Foltz, C. B., et al. 1991, ApJ, 373, 465

Francis, P. J., Whiting, M. T., \& Webster, R. L. 2000, Publ. Astron. Soc. Aust., 53, 56

Green, P. J. 1996, ApJ, 467, 61

Hagen, H.-J., Engels, D., \& Reimers, D. 1999, A\&AS, 134, 483

Hartwick, F. D. A., \& Schade, D. 1990, ARA\&A, 28, 437

Hawkins, M. R. S. 1983, MNRAS, 202, 571

Hook, I. M., McMahon, R. G., Boyle, B. J., \& Irwin, M. J. 1994, MNRAS, 268, 305

Kembhavi, A. K., \& Narlikar, J. V. 1999, Quasars and Active Galactic Nuclei (Cambridge: University Press)

Kim, D.-W., \& Elvis, M. 1999, ApJ, 516, 9

La Franca, F., Cristiani, S., \& Barbieri, C. 1992, AJ, 103, 1062

Ledden, S. E., \& O’Dell, S. L. 1983, ApJ, 270, 434

Lehmann, H., Ziener, R., Ball, M., \& Pitz, E. 1995, in ASP Conf. Ser. 71, Tridimensional Optical Spectroscopic Methods in Astrophysics, ed. G. Comte, \& M. Marcelin, 219

Lira, P., Lawrence, A., \& O'Brien, P., et al. 1999, MNRAS, 305,109 
Majewski, S. R., Munn, J. A., \& Kron, R. G., et al. 1991, in ASP Conf. Ser. 21, The Space Distribition of Quasars, ed. D. Crampton, 55

Masci, F. J., Webster, R. L., \& Francis, P. J. 1998, MNRAS, 301,975

Masci, F. J., Drinkwater, M. J., \& Webster, R. L. 1999, ApJ, 510,703

McDowell, J. C., Canizares, C., \& Elvis, M., et al. 1995, ApJ, 450,585

Meusinger, H., Klose, S., Ziener, R., \& Scholz, R.-D. 1995, in ASP Conf. Ser. 84, The Future Utilisation of Schmidt Telescopes, ed. J. M. Chapman, et al., 486

Meusinger, H., Ball, M., Scholz, R.-D., \& Irwin, M. 1997, Astron. Ges. Abstract Ser., 13, 233

Meusinger, H., Scholz, R.-D., Irwin, M., et al. 1998, Astron. Ges. Abstract Ser., 14, 109

Meusinger, H., Brunzendorf, J., Scholz, R.-D., \& Irwin, M. 1999, in Treasure Hunting in Astronomical Plate Archives, ed. P. Kroll et al. (Thun \& Frankfurt: Verlag Harri Deutsch), 122

Meusinger, H., \& Brunzendorf, J. 2000, Astron. Ges. Abstr. Ser., 17,86

Meusinger, H., \& Brunzendorf, J. 2001, Information Bulletin on Variable Stars, 5035, 1

Moran, E. C., Filippenko, A. V., Ho, L. C., et al. 1999, PASP, 111,969

Najita, J., Dey, A., \& Brotherton, M. 2000, AJ, 120, 2859

Nakanishi, K., Akiyama, M., Ohta, K., \& Yamada, T. 2000, ApJ, 534, 587

Puchnarewicz, E. M., Mason, K. O., Carrera, F. J., et al. 1997, MNRAS, 291, 177

Rieke, G. H., Lebofsky, M. J., \& Wisniewski, W. A. 1982, ApJ, 263,73
ROSAT Consortium 2000, The Second ROSAT Source Catalog of Pointed Observations, ROSAT News 72

Scholz, R.-D., Meusinger, H., \& Irwin, M. 1997, A\&A, 325, 457

Skrutskie, M. F., Schneider, S. E., Stiening, R., et al. 1997, in The Impact of Large Scale Near-IR Sky Surveys, ed. F. Garzon et al. (Dordrecht: Kluwer Academic Publishing Company), 25

Smith, H. E., \& Spinrad, H. 1980, ApJ, 236, 419

Srianand, R., \& Kembhavi, A. 1997, ApJ, 478, 70

Stickel, M., Rieke, G. H., Kühr, H., \& Rieke, M. J. 1996, ApJ, 468,556

Turnshek, D. A. 1988, in QSO Absorption Lines: Probing the Universe, ed. J. C. Blades, et al. (Cambridge: University Press), 17

Véron, P. 1993, in Proceedings of the 31st Liège international astrophysical colloquium, 237

Véron-Cetty, M. P., Véron, P., \& Tarenghi, M. 1983, A\&A, 119,69

Véron-Cetty, M. P., \& Véron, P. 1998, Quasars and Active Galactic Nuclei, 8th Ed., ESO Scientific Report, 18, 1

Véron-Cetty, M. P., \& Véron, P. 2000, Quasars and Active Galactic Nuclei, 9th Ed., ESO Scientific Report, 19, 1

Vignali, C., Mignoli, M., Comastri, A., Maiolino, R., \& Fiore, F. 2000, MNRAS, 314, L11

Webster, R. L., Francis, P. J., Peterson, B. A., Drinkwater, M. J., \& Masci, F. J. 1995, Nature, 375, 469

White, R. L., Becker, R. H., Helfand, D. J., \& Gregg, M. D. 1997, ApJ, 475, 479

Wisotzki, L. 2000, A\&A, 353, 853

Zheng, W., Kriss, G. A., Telfer, R. C., Grimes, J. P., \& Davidsen, A. F. 1997, ApJ, 475, 469 